\title{
Recurrent Stupor Due to Lysinuric Protein Intolerance
}

\author{
N. V. Ahsan Moosa, D. Minal, Ananth N Rao, * Anand Kumar \\ Departments of Neurology \& *Biochemistry, Amrita Institute of Medical Sciences \& Research Center, Cochin, Kerala, India
}

\begin{abstract}
Recurrent stupor in children is an uncommon clinical problem with a wide differential diagnosis; inherited metabolic disorders account for a vast majority. We report a 9-yearold girl with recurrent episodes of stupor. Initial episode was treated as viral encephalitis and the second episode was managed as non-convulsive status epilepticus. Hyperammonemia was detected in the last episode. Metabolic work-up after dietary protein challenge revealed classical biochemical features of lysinuric protein intolerance. She was managed with protein-restricted diet, which resulted in marked neurological improvement. LPI is a rare inherited metabolic disorder due to membrane transport defect of cationic amino acids.
\end{abstract}

Key words: Lysinuric protein intolerance, hyperammonemia, recurrent stupor.

\section{Introduction}

Recurrent stupor in pediatric age group presents a diagnostic challenge to the physician. Inherited metabolic disorders [IMD] such as organic acidemias, urea cycle disorders, conditions resulting in recurrent hypoglycemia and fatty acid oxidation defects account for the majority of cases of recurrent stupor. ${ }^{1}$ Sophisticated investigations may be required to arrive at the diagnosis. Early diagnosis may significantly improve the outcome, and in a few, may even prove to be life saving.

We report a case of a 9-year-old-girl with recurrent episodes of stupor due to lysinuric protein intolerance [LPI], a rare hyperammonemic disorder. To our knowledge this is the first reported case from India.

\section{Case report}

A 9-year-old-girl was referred to us for evaluation of recurrent stupor. She was born of consanguineous parentage and had an uneventful perinatal period. Her developmental milestones were normal till sixth year. At age 6 , she developed recurrent vomiting, altered sensorium, convulsions and was comatose on day 3 of illness. She had no fever, rash or other systemic symptoms. Cerebrospinal fluid analysis and MRI brain were normal. She was treated as a case of viral encephalitis with acyclovir and anti-convulsants. She improved over 1 to 2 weeks.

A year later she had another episode of stupor. A diagnosis of non-convulsive status epilepticus was made based on high amplitude delta slowing on electroencephalogram. She improved over 3 days. Third episode occurred at 9 year of age with recurrent vomiting and drowsiness requiring hospitalization. On admission to hospital, she was drowsy. She was $118 \mathrm{cms}$ tall, weighed $19 \mathrm{kgs}$, and head circumference was 48 cms. Fundus and cranial nerve examination was unremarkable. Mild spasticity in all four limbs with hyper-reflexia and bilateral extensor plantar responses were noted. Examination of other systems were unremarkable.

A detailed dietary history revealed aversion to high protein diet such as milk, fish, eggs and pulses. Consumption of these food items often caused repeated vomiting. Child's mother reported that the recent episode followed intake of high protein diet. she could not reliably recall any specific diet related worsening in the initial episodes. From the age of 6 , she has been noted to have a clumsy gait with frequent falls along with poor scholastic performance and hyperactivity.

Biochemical evaluation including blood glucose, serum sodium, potassium, urea, creatinine, SGOT, SGPT, lactate, arterial blood gas analysis and bicarbonates levels were normal. MRI brain was normal. EEG revealed diffuse slowing. Serum ammonia was 125 micromoles/liter [normal, 30-65 micromoles/liter] on second day of illness. She gradually improved with protein restriction, oral lactulose and sodium benzoate therapy and was alert in a week.

Recurrent encephalopathy, hyperammonemia, diet related worsening and aversion to high protein diet strongly suggested a possibility of disorder resulting in hyperammonemia. In view of her diet related symptoms, She was fed $4 \mathrm{gm} / \mathrm{kg}$ of protein 
as two divided meals. Arterial blood samples were drawn after overnight fasting and 2 hours after first test meal and 2 and 4 hours after second test meal; urine was collected simultaneously. Serial samples showed progressive rise in ammonia- 50, 70, 80 and 106 micro mol/liter respectively. She became drowsy after second meal and vomited few times. Serum amino acid pattern by partition chromatography showed subnormal levels of lysine, arginine and ornithine. Marked lysinuria with elevated urinary excretion of ornithine, arginine and orotic acid was detected. (Table-1) Serum citrulline and glutamine were 78 micro mol/l (normal 10-30) and 1043 micro mol/l (normal 330-650) respectively. Organic acid assay was normal. A diagnosis of lysinuric protein intolerance was made. High resolution CT of lungs showed no evidence of interstitial lung disease; 24-hour urine protein estimation was normal. Bone densitometry revealed borderline osteoporosis.

One month after protein-restricted diet, she had significant improvement in gait with less falls and also had striking improvement in attention; subtle pyramidal signs persisted.

\section{Discussion}

Lysinuric protein intolerance is a rare IMD resulting in recurrent hyperammonemia. ${ }^{[1]}$ LPI is a multi-system disorder, with episodic hyperammonemia being the characteristic manifestation. ${ }^{[1,2,3]}$

Children with LPI are usually symptom free while on breastfeeds, as breast milk is relatively low in lysine. ${ }^{[1]}$ While being weaned, they develop vomiting, diarrhea, aversion to proteinrich food, and consequently failure to thrive. The course of LPI is punctuated by acute exacerbations of hyperammonemia often due to high protein food items. ${ }^{[1]}$ Hyperammonemia is caused by the lack of sufficient ornithine to support the activity of ornithine transcarbamylase (OTC), a urea-cycle enzyme in liver. ${ }^{[1,3]}$ Elevated citrulline levels and characteristic changes of dibasic amino acid profile in urine and serum differentiate LPI form OTC deficiency. Mild episodes of postprandial hyperammonemia may go unrecognized. Our case had clinical and biochemical features of LPI. This is an autosomal recessive disorder due to mutation in SLC7A7, (for solute carrier family 7 , member 7 ), a gene encoding a putative permease-related protein. ${ }^{[2]}$ This results in defective transport of
Table 1: Amino acid levels in serum and urine samples collected 2 hours after test meal.

\begin{tabular}{|c|c|c|}
\hline Amino acid & Serum & Urine \\
\hline Lysine & 68 (91-170 micro $\mathrm{mol} / \mathrm{l})$ & 987 (89-602 micro mol/g creatinine \\
\hline Alanine & 328 (212-407 micro mol /L) & $\begin{array}{l}896 \text { ( } 151-814 \text { micro } \mathrm{mol} / \mathrm{g} \\
\text { creatinine) }\end{array}$ \\
\hline Ornithine & 63 (92-194 micro mol /L) & 69 (0-62 micro mol /g creatinine) \\
\hline Arginine & 24 (27-91 micro mol /L) & 81 (0-62 micro mol /g creatinine) \\
\hline
\end{tabular}

dibasic amino acids namely lysine, arginine and ornithine in renal tubules, intestine and hepatocytes, leading to decreased absorption and excessive loss.

Prevention of postprandial hyperammonemia by dietary protein restriction forms the main stay of treatment for LPI. ${ }^{1}$ Other measures to reduce ammonia level may be needed during crisis. ${ }^{[4]}$ Citrulline and low dose oral lysine have been tried with variable success. ${ }^{[1,5,6]}$ Neurological manifestations in our patient responded partially to dietary management alone. ${ }^{[1]}$ Periodic monitoring is required for early detection of renal or pulmonary involvement.

In conclusion, recurrent stupor warrants careful evaluation for inherited metabolic disorders. A good dietary history and appropriate investigations is crucial for arriving at the correct diagnosis of LPI.

\section{References}

1. Simell O. Lysinuric protein intolerance and other cationic aminoacidurias. In: Scriver CR, Beaudet AL, Sly Ws, Valle D. editors. The metabolic and molecular bases of inherited disease. 8 th ed. New York, McGraw-Hill Medical Publication division, 2001, Volume 3, p 4933-56

2. Borsani G, Bassi MT, Sperandeo MP, De Grandi A, Buoninconti A, Riboni M, et al. SLC7A7, encoding a putative permease-related protein, is mutated in patients with lysinuric protein intolerance. Nat Genet 1999;21:297-301.

3. Palacin M, Bertran J, Chillaron J, Estevez R, Zorzano A. Lysinuric protein intolerance: mechanisms of pathophysiology. Mol Genet Metab 2004; $81 \mathrm{Suppl}$ 1:S27-37

4. Brusilow SW, Danney M, Waber LJ, Batshaw M, Burton B, Levitsky L, et al. Treatment of episodic hyperammonemia in children with inborn errors of urea synthesis. New Eng J Med 1984;310:1630-4.

5. Carpenter TO, Levy HL, Holtrop ME, Shih VE, Anast CS. Lvsinuric protein intolerance presenting as childhood osteoporosis: clinical and skeletal response to citrulline therapy. New Eng .J Med 1985;312:290-4.

6. Lukkarinen M, Nanto-Salonen K, Pulkki K, Aalto M, Simell O. Oral supplementation corrects plasma lysine concentrations in lysinuric protein intolerance Metabolism, 2003:52:935-8.

Accepted on 06-05-2005 